\title{
New Scheme Transformations and Application to Study Scheme Dependence of an Infrared Zero of the Beta Function in Gauge Theories
}

\author{
Gongjun Choi and Robert Shrock \\ C. N. Yang Institute for Theoretical Physics \\ Stony Brook University \\ Stony Brook, NY 11794
}

\begin{abstract}
We present two new one-parameter families of scheme transformations and apply these to study the scheme dependence of the infrared zero in the beta function of an asymptotically free nonAbelian gauge theory up to four-loop order. Our results provide a further quantitative measure of this scheme dependence, showing that for moderate values of the gauge coupling and the parameter specifying the scheme transformation, this dependence is relatively mild. We also remark on a generalized multi-parameter family of rational scheme transformations.
\end{abstract}

PACS numbers: 11.10.Hi,11.15.-q,11.15.Bt

\section{INTRODUCTION}

The dependence of the interaction coupling of a quantum field theory on the Euclidean momentum scale, $\mu$, where it is measured, is of fundamental importance. This dependence is described by the renormalization-group beta function of the theory [1]. In particular, it is of interest to study the evolution of the running gauge coupling $g \equiv g(\mu)$ of an asymptotically free gauge theory from the deep ultraviolet (UV) region at large $\mu$, where it is small, to the infrared (IR) region at small $\mu$. Let us consider such a theory (in $d=4$ spacetime dimensions) with a non-Abelian gauge group $G$ and $N_{f}$ massless fermions in a given representation $R$ of $G$. If the beta function of this theory has a zero at a value $\alpha_{I R}$, where $\alpha=g^{2} /(4 \pi)$, then, as the scale $\mu$ decreases from large values, the coupling evolves toward $\alpha_{I R}$, which is thus an exact or approximate infrared fixed point (IRFP) of the renormalization group.

The perturbative calculation of the value of $\alpha_{I R}$ at $\ell$ loop order is complicated by the property that the terms in the beta function with $\ell \geq 3$ depend on the scheme used for the regularization and renormalization of the theory. The presence of scheme dependence in higherloop calculations is, of course, a general property of quantum field theory; here we focus on its effects on $\alpha_{I R}$. It is important to determine how sensitively $\alpha_{I R}$ depends on the scheme used for its calculation. To do this, one can compute the beta function in one scheme, then carry out a transformation to a different scheme, and compare the respective values of the IR zero of the beta functions in these schemes. A useful general framework is provided by dimensional regularization of the Feynman integrals involved in loop calculations [2]. An early scheme used dimensional regularization combined with minimal subtraction of the poles at $d=4$ in the Euler $\Gamma$ functions resulting from the Feynman integrals [3], and this was extended to the widely used modified minimal subtraction $(\overline{\mathrm{MS}})$ scheme with the subtraction of certain associated constants in the Taylor-Laurent expansion of these $\Gamma$ functions [4]. There has long been interest in studying various scheme transformations to reduce higher-order corrections in perturbative calculations in quantum chromodyamics (QCD) (e.g., 5]-7] ). In QCD, one studies the effect of applying these scheme transformations in the vicinity of the UV zero of the beta function at $\alpha=0$, the UV fixed point (UVFP) of QCD.

The situation is significantly different when one studies an IR zero of the beta function away from the origin, $\alpha=0$. Refs. [8, 9] pointed out that there is much less freedom in constructing and applying acceptable scheme transformations at an IR zero than there is at the UVFP at $\alpha=0$ and gave examples of several scheme transformations that are perfectly acceptable in the vicinity of the UVFP at $\alpha=0$ in an asymptotically free theory but exhibit unphysical, pathological properties, when applied at a generic IRFP away from the origin. Further studies of scheme transformations and their application to an IRFP and IR properties of an asymptotically free gauge theory have been carried out in [10]-[14]. In addition to a variety of transformations to different schemes starting from the $\overline{\mathrm{MS}}$ scheme [4] studied in [8 11], these have included comparisons of results for the IRFP and IR properties in the $\overline{\mathrm{MS}}$ scheme with results obtained with the modified regularization-invariant, $\mathrm{RI}^{\prime}$ scheme, and the minimal momentum (MOM) subtraction scheme 12 14].

In this paper we will construct and study two new oneparameter families of scheme transformations, which we denote as $S_{L_{r}}$ and $S_{Q_{r}}$, where the subscript $r$ is the respective parameter on which each transformation depends. We show that these scheme transformations satisfy the rather restrictive set of conditions set forth in [8, 9] to be physically acceptable at an IR zero of the beta function at moderate coupling. Having done this, we then apply them to study further the sensitivity of the IR zero of the $\ell$-loop beta function of asymptotically free vectorial non-Abelian gauge theories. Our results provide a further quantitative measure of the scheme dependence of the value of an IRFP and show that for moderate values of $\alpha_{I R}$, as calculated at the $\ell$-loop level with $\ell$ up to four loops, this dependence is relatively mild. 
This paper is organized as follows. In Sect. II we discuss some relevant background and the basic properties of scheme transformations. In Sect. III we present a new one-parameter family of scheme transformations denoted $S_{L_{r}}$ and apply it to analyze the scheme dependence of the IR zero of the beta function in an asymptotically free non-Abelian gauge theory up to four-loop order. In Sect. IV] we introduce a general class of multi-parameter rational scheme transformations, denoted $S_{[p, q]}$, and in Sect. $\mathrm{V}$ we analyze a one-parameter family that is a member of this class, namely $S_{[0,1]} \equiv S_{Q_{r}}$, and again apply this to study the scheme dependence of an IR zero of an asymptotically free gauge theory. Sect. VI contains a comparison of some general features of these scheme transformations with the $S_{\mathrm{sh}_{\mathrm{r}}}$ scheme transformations previously studied in [8]-[11] involving a sinh transformation function. Some remarks on other $S_{[p, q]}$ families of scheme transformations are given in Sect. VII. Section VIII contains some remarks on IR-free theories. Our conclusions are given in Sect. IX. Certain auxiliary results are listed in an Appendix.

\section{BACKGROUND AND METHODS}

\section{A. Beta Function}

In this section we discuss some relevant background. We define $a(\mu)=a$ as

$$
a \equiv \frac{g^{2}}{16 \pi^{2}}=\frac{\alpha}{4 \pi},
$$

where here and below, the argument $\mu$ will often be suppressed in the notation. The beta function is $\beta_{g}=d g / d t$ or equivalently,

$$
\beta_{\alpha} \equiv \frac{d \alpha}{d t}=\frac{g}{2 \pi} \beta_{g}
$$

where $d t=d \ln \mu$. The function $\beta_{\alpha}$ has the series expansion

$$
\beta_{\alpha}=-2 \alpha \sum_{\ell=1}^{\infty} b_{\ell} a^{\ell}=-2 \alpha \sum_{\ell=1}^{\infty} \bar{b}_{\ell} \alpha^{\ell}
$$

where $\bar{b}_{\ell}=b_{\ell} /(4 \pi)^{\ell}$. The $n$-loop $(n \ell)$ beta function, denoted $\beta_{\alpha, n \ell}$, is obtained from Eq. (2.3) by replacing the upper limit on the $\ell$ loop summation by $n$ instead of $\infty$. The $b_{\ell}$ for $\ell=1,2$ are independent of the scheme used for regularization and renormalization, while $b_{\ell}$ with $\ell \geq 3$ are scheme-dependent [16]. For a non-Abelian gauge theory, the coefficients $b_{1}$ and $b_{2}$ were calculated in [17] and [18], while $b_{3}$ and $b_{4}$ were calculated in the MS scheme in [19] and 20]. We denote the IR zero of the $n$-loop beta function $\beta_{\alpha, n \ell}$ as $\alpha_{I R, n \ell}=4 \pi a_{I R, n \ell}$.

\section{B. Scheme Transformations}

A scheme transformation can be expressed as a mapping between $\alpha$ and $\alpha^{\prime}$ or equivalently, between $a$ and $a^{\prime}$, namely

$$
a=a^{\prime} f\left(a^{\prime}\right) .
$$

In the limit where $a$ and $a^{\prime}$ vanish, the theory becomes free, so a scheme transformation has no effect. This implies the condition $f(0)=1$. The functions $f\left(a^{\prime}\right)$ that we consider have Taylor series expansions about $a=a^{\prime}=0$ of the form

$$
f\left(a^{\prime}\right)=1+\sum_{s=1}^{s_{\max }} k_{s}\left(a^{\prime}\right)^{s}
$$

where the $k_{s}$ are constants, and $s_{\max }$ may be finite or infinite. Given the form (2.5), it follows that the Jacobian

$$
J=\frac{d a}{d a^{\prime}}=\frac{d \alpha}{d \alpha^{\prime}}
$$

has the series expansion

$$
J=1+\sum_{s=1}^{s_{\max }}(s+1) k_{s}\left(a^{\prime}\right)^{s}
$$

and thus satisfies

$$
J=1 \quad \text { at } \quad a=a^{\prime}=0 .
$$

The beta function in the transformed scheme is

$$
\beta_{\alpha^{\prime}} \equiv \frac{d \alpha^{\prime}}{d t}=\frac{d \alpha^{\prime}}{d \alpha} \frac{d \alpha}{d t}=J^{-1} \beta_{\alpha}
$$

with the series expansion

$$
\beta_{\alpha^{\prime}}=-2 \alpha^{\prime} \sum_{\ell=1}^{\infty} b_{\ell}^{\prime}\left(a^{\prime}\right)^{\ell}=-2 \alpha^{\prime} \sum_{\ell=1}^{\infty} \bar{b}_{\ell}^{\prime}\left(\alpha^{\prime}\right)^{\ell},
$$

where $\bar{b}_{\ell}^{\prime}=b_{\ell}^{\prime} /(4 \pi)^{\ell}$. Since Eqs. (2.9) and (2.10) define the same function, one can solve for the $b_{\ell}^{\prime}$ in terms of the $b_{\ell}$ and $k_{s}$. This yields the results $b_{1}^{\prime}=b_{1}$ and $b_{2}^{\prime}=b_{2}$. In [8, 9], explicit expressions were calculated for higherloop $b_{\ell}^{\prime}$ with $\ell \geq 3$ in terms of the $b_{\ell}$ and $k_{s}$. In general, it was shown that the coefficient $b_{\ell}^{\prime}$ with $\ell \geq 3$ in the transformed scheme is a linear combination of $b_{n}$ with $1 \leq n \leq \ell$ with coefficients that are algebraic functions of the various $k_{s}$. Some relevant results are given in the Appendix.

Given that the $b_{\ell}$ for $\ell \geq 3$ are scheme-dependent, one may ask whether it is possible to transform to a scheme in which the $b_{\ell}^{\prime}$ are all zero for $\ell \geq 3$, i.e., a scheme in which the two-loop $\beta$ function is exact. Near the UV fixed point at $\alpha=0$, this is possible, as emphasized by 't Hooft 21]. The resultant scheme, in which the beta function truncates at two-loop order is commonly called the 't Hooft scheme [22]. 
Ref. [9] presented an explicit scheme transformation which, starting from an arbitrary scheme, transforms to the 't Hooft scheme. This necessarily has $s_{\max }=\infty$. However, Refs. [9, 10] also noted that although this scheme transformation is acceptable in the vicinity of a zero of the beta function at $\alpha=0$ (UV zero for an asymptotically free theory or IR zero for an infrared-free theory), it cannot, in general, be applied to a generic zero of the beta function (IR zero of an asymptotically free theory or UV zero of an infrared-free theory) away from $\alpha=0$. Ref. [11] constructed and studied a one-parameter class of scheme transformations, denoted $S_{R, m, k_{1}}$ having $s_{\max }=m \geq 2$, with the property that an $S_{R, m, k_{1}}$ scheme transformation eliminates the $\ell$-loop terms in the beta function of a gauge theory from loop order $\ell=3$ to order $\ell=m+1$, inclusive and can be applied not only at a zero of the beta function at $\alpha=0$ but also for a zero of the beta function away from $\alpha=0$.

In order to be physically acceptable, a scheme transformation must satisfy several conditions, as was discussed in [9]. We state these for an asymptotically free gauge theory: (i) condition $C_{1}$ : the scheme transformation must map a real positive $\alpha$ to a real positive $\alpha^{\prime}$; (ii) $C_{2}$ : the scheme transformation should not map a moderate value of $\alpha$, for which perturbation theory may be reliable, to a value of $\alpha^{\prime}$ that is so large that perturbation theory is unreliable, or vice versa; (iii) $C_{3}$ : the Jacobian $J$ should not vanish (or diverge) or else the transformation would be singular; and (iv) $C_{4}$ : since the existence of an IR zero of $\beta$ is a scheme-independent property of an theory, a scheme transformation must satisfy the condition that $\beta_{\alpha}$ has an IR zero if and only if $\beta_{\alpha^{\prime}}$ has an IR zero. Since $J=1$ for $a=a^{\prime}=0$, a corollary of condition $C_{3}$ is that $J$ must be positive. Since one can define a transformation from $\alpha$ to $\alpha^{\prime}$ and the inverse from $\alpha^{\prime}$ to $\alpha$, these conditions apply going in both directions. In passing, we note that with obvious changes (IR zero $\rightarrow$ possible UV zero in condition $C_{4}$ ), these conditions also apply to to an infrared-free gauge theory such as $\mathrm{U}(1)$ and a non-Abelian gauge theory with sufficiently many fermions, as discussed in 23], and to an (infrared-free) scalar theory, such as an $\mathrm{O}(N) \lambda|\vec{\phi}|^{4}$ theory, as analyzed in [24].

These four conditions $C_{1}-C_{4}$ can always be satisfied by scheme transformations used to study the UV fixed point in an asymptotically free theory. However, as was pointed out in [8] and shown with a number of examples in [8]-[11], they are not automatically satisfied, and indeed, are quite restrictive conditions when one applies the scheme transformation at a zero of the beta function away from the origin, $\alpha=0$, i.e., at an IR zero of the beta function for an asymptotically free theory or a possible UV zero of the beta function for an infrared-free theory. For example, recall the scheme transformation denoted $S_{\mathrm{th}_{\mathrm{r}}}$ [8, 9], defined by $a=(1 / r) \tanh \left(r a^{\prime}\right)$, depending on a parameter $r$. Since this transformation is an even function of $r$, one may take $r \geq 0$ without loss of generality. The $S_{\mathrm{th}_{\mathrm{r}}}$ transformation is well-behaved near the UVFP at $a=a^{\prime}=0$ in an asymptotically free theory, but is not acceptable at a generic IR zero of the beta function. The reason is evident from its inverse, $a^{\prime}=(2 r)^{-1} \ln [(1+r a) /(1-r a)]$. As $r a$ approaches 1 from below, $a^{\prime} \rightarrow \infty$, and for $r a>1, a^{\prime}$ is complex. Hence, this transformation violates conditions $C_{1}, C_{2}$, and $C_{4}$. For example, for $r=4 \pi$, this scheme transformation is $\alpha=\tanh \alpha^{\prime}$ and the inverse is $\alpha^{\prime}=(1 / 2) \ln [(1+\alpha) /(1-\alpha)]$, with the pathologies occurring as $\alpha$ approaches 1 from below. For $r=8 \pi$, the pathologies occur as $\alpha$ approaches the value 0.5 from below. As this example and the others analyzed in [8]-[1] show, the construction and application of a physically acceptable scheme transformation at a zero of the beta function away from the origin is considerably more difficult than at a zero of the beta function at the origin, as in scheme transformations used in QCD 7].

In the following, to avoid overly complicated notation, we will use the generic notation $\alpha^{\prime}$ for the result of the application of each scheme transformation to an initial $\alpha$, with it being understood that this refers to the specific transformation under consideration. Where it is necessary for clarity, we will use a subscript to identify the specific scheme $S$ being discussed.

\section{UV to IR Evolution of Non-Abelian Gauge Theories}

Since we will apply our new scheme transformations to study the scheme dependence of an IR zero of the beta function for a vectorial, asymptotically free (nonAbelian) gauge theory, it is appropriate to review briefly some of the properties of this theory. Let us consider such a theory with gauge group $G$ and $N_{f}$ massless fermions transforming according to a representation $R$ of $G$. Our assumption of massless fermions does not entail any significant loss of generality, since if a given fermion had a mass $m$, then in the UV to IR evolution of the theory, as the reference Euclidean momentum scale $\mu$ decreased past $m$, one would integrate out this fermion to construct the low-energy effective field theory applicable at scales $\mu<m$, so the further evolution into the IR would be essentially equivalent to a theory without this massive fermion present. With the minus sign extracted in Eq. (2.3), the asymptotic freedom of the theory means that the one-loop coefficient $b_{1}$ in Eq. (2.3) is positive. As $N_{f}$ increases, $b_{1}$ decreases and eventually would vanish at $N_{f, b 1 z}=11 C_{A} /\left(4 T_{f}\right)[25,26]$. Thus, the asymptotic freedom yields an upper bound on $N_{f}$, namely, $N_{f}<N_{f, b 1 z}$.

For small $N_{f}$, the two-loop coefficient $b_{2}$ has the same positive sign as $b_{1}$, so the (perturbatively calculated) twoloop beta function, $\beta_{\alpha, 2 \ell}$, has no IR zero. The coefficient $b_{2}$ decreases as $N_{f}$ increases and passes through zero to negative values as $N_{f}$ ascends through the value

$$
N_{f, b 2 z}=\frac{17 C_{A}^{2}}{2 T_{f}\left(5 C_{A}+3 C_{f}\right)} .
$$


Since $N_{f, b 2 z}<N_{f, b 1 z}$, there is an interval of values of $N_{f}$, denoted $I$, given by

$$
I: \quad N_{f, b 2 z}<N_{f}<N_{f, b 1 z},
$$

in which the two-loop beta function has an IR zero. This occurs at $a_{I R, 2 \ell}=-b_{1} / b_{2}$, i.e.

$$
\alpha_{I R, 2 \ell}=-\frac{4 \pi b_{1}}{b_{2}},
$$

which is physical for $b_{2}<0$ [18, 27]. The scheme independence of $b_{1}$ and $b_{2}$ implies that $\alpha_{I R, 2 \ell}$ is also schemeindependent. Since an IR zero of $\beta_{\alpha, n \ell}$ for $n \geq 3$ depends on the scheme $S$ used for the computation, we denote it here as $\alpha_{I R, n \ell, S}$.

Let us assume $N_{f} \in I$, so that $\beta_{\alpha, 2 \ell}$ has an IR zero, $\alpha_{I R, 2 \ell}$. If $N_{f}$ is close to $N_{f, b 1 z}$, then, as noted, $\alpha_{I R, 2 \ell}$ is small. In this case, one expects that the UV to IR evolution of the theory leads to a deconfined non-Abelian Coulomb phase without any spontaneous chiral symmetry breaking [27]. In this case, the IR zero is an exact IRFP. As $N_{f}$ decreases, $\alpha_{I R, 2 \ell}$ increases. If $\alpha_{I R, 2 \ell}$ is sufficiently large, the UV to IR evolution generically leads to the formation of bilinear fermion condensates in the most attractive channel, with attendant spontaneous chiral symmetry breaking and dynamical generation of effective masses for the fermions involved. In the ladder approximation to the Schwinger-Dyson equation for the fermion propagator, this occurs as $\alpha$ increases through a value $\alpha_{c r}$ given by 28] $\alpha_{c r}=\pi /\left(3 C_{f}\right)$. Taking account of the intrinsic uncertainties involved in the strongly coupled physics of fermion condensate formation, one may infer more generally that the actual critical value of $\alpha$ is expected to satisfy $\alpha_{c r} C_{f} \sim O(1)$. The fermions involved in the condensate gain dynamical masses of order the chiral-symmetry-breaking scale and are integrated out of the low-energy effective field theory below this scale. Thus, the beta function changes to one with the effective $N_{f}=0$, which does not have an IR zero, and hence the gauge coupling increases, eventually exceeding the range where perturbative calculations are applicable. In this case, the IR zero is only an approximate IRFP of the renormalization group. One defines a critical value, $N_{f, c r}$, that separates the two types of UV to IR evolution; for $N_{f}>N_{f, c r}$, this evolution is to a massless non-Abelian Coulomb phase, while for $N_{f}<N_{f, c r}$, it involves the above-mentioned chiral symmetry breaking.

As $N_{f}$ decreases toward $N_{f, c r}$, the resultant IR zero occurs at moderately strong coupling, and consequently it is necessary to go beyond the two-loop level and calculate $\alpha_{I R, n \ell}$ at higher loop order [29]. This was done up to four-loop order for $\alpha_{I R, n \ell}$ and for the anomalous dimension, $\gamma_{m}$, of the fermion bilinear for a general gauge group and fermion representation in [30, 31]. For fermions in the fundamental representation, it was found that, in the $\overline{\mathrm{MS}}$ scheme, relative to the (scheme-independent) twoloop value, $\alpha_{I R, 2 \ell}$.

$$
\alpha_{I R, 3 \ell, \overline{\mathrm{MS}}}<\alpha_{I R, 4 \ell, \overline{\mathrm{MS}}}<\alpha_{I R, 2 \ell} .
$$

The shifts in the value of the IR zero with ascending loop order were found to become smaller as $N_{f}$ approaches $N_{f, b 1 z}$. Comparisons were made with the extensive lattice studies of this physics for various gauge groups and fermion representations [32]. Further higher-loop results on structural properties of $\beta$ and application to the IRFP were calculated in [33]-34]. Because the coefficients $b_{\ell}$ for $\ell \geq 3$ are scheme-dependent, these higher-loop calculations naturally led to the study of scheme-dependence in [8]- 14]. In the region of $N_{f}$ slightly less than $N_{f, c r}$, where the theory confines but behaves in a quasi-scaleinvariant manner over an extended interval in $\mu$, some insight has been gained from continuum studies of the changes in the spectrum of gauge-singlet hadrons as compared with the spectrum in a QCD-like theory [28, 35]. Intensive research on this region exhibiting quasi-scaleinvariant behavior has also considerably deepened one's knowledge of this physics [32].

Let us consider a well-behaved (family of) scheme transformation(s) $S_{\{r\}}$ where in this paragraph, $\{r\}$ symbolizes a set of one or more parameters, such that $S_{\{0\}}$ is the identity. It follows that if one applies the transformation $S_{\{r\}}$ to the $\overline{\mathrm{MS}}$ scheme, then, for a given loop order $n$,

$$
\lim _{\{r\} \rightarrow\{0\}} \alpha_{I R, n \ell, S_{\{r\}}}^{\prime}=\alpha_{I R, n \ell, \overline{\mathrm{MS}}} .
$$

Furthermore, since the IR zero in $\beta_{\alpha, n \ell}$ approaches zero as $N_{f}$ approaches $N_{f, b 1 z}$ from below, one has the formal result that, with $N_{f}$ extended from a nonnegative integer variable to a nonnegative real variable,

$$
\lim _{N_{f} \nearrow N_{f, b 1 z}} \alpha_{I R, n \ell, S_{\{r\}}}=\lim _{N_{f} \nearrow N_{f, b 1 z}} \alpha_{I R, n \ell, \overline{\mathrm{MS}}}=0 .
$$

Moreover, if the set of parameters $\{r\}$ specifying the scheme transformation is such that this transformation is sufficiently close to the identity, then it preserves the relative order of the values of the IR zeros of the $n$-loop beta function. We recall that for fermions in the fundamental representation, in the $\overline{\mathrm{MS}}$ scheme, the three-loop and four-loop values of the IR zero are in the order given by Eq. (2.14) above.

\section{THE $S_{L_{r}}$ SCHEME TRANSFORMATION}

Here we introduce and study a scheme transformation, denoted $S_{L_{r}}$, where $L$ stands for logarithm and $r$ for the parameter on which a transformation in this family depends. This is thus actually a one-parameter family of scheme transformations. We show that the $S_{L_{r}}$ scheme transformation satisfies the necessary conditions to be acceptable at a zero of the beta function away from the origin, for a reasonable range of $|r|$, and we then apply it to the calculation, at higher-loop order, of an IR zero of the beta function for an asymptotically free non-Abelian gauge theory. This calculation provides a measure of the scheme dependence of the value of this IR zero. 
The $S_{L_{r}}$ scheme transformation is defined as

$$
S_{L_{r}}: \quad a=\frac{\ln \left(1+r a^{\prime}\right)}{r},
$$

where $r$ is a (real) parameter. Writing Eq. (3.1) in the form of Eq. (2.4), the transformation function is

$$
S_{L_{r}}: \quad f\left(a^{\prime}\right)=\frac{\ln \left(1+r a^{\prime}\right)}{r a^{\prime}} .
$$

This transformation function satisfies

$$
\lim _{a^{\prime} \rightarrow 0} f\left(a^{\prime}\right)=1
$$

in accordance with the requirement that $f(0)=1$. Note also that

$$
\lim _{r \rightarrow 0} f\left(a^{\prime}\right)=1
$$

where the limit may be taken through either positive or negative values of $r$. The scheme transformation (3.1) has the inverse

$$
a^{\prime}=\frac{e^{r a}-1}{r}
$$

The Jacobian $J=d a / d a^{\prime}$ is

$$
J=\frac{1}{1+r a^{\prime}}=e^{-r a}
$$

The transformation function $f\left(a^{\prime}\right)$ has the Taylor series expansion

$$
f\left(a^{\prime}\right)=1+\sum_{s=1}^{\infty} \frac{\left(-r a^{\prime}\right)^{s}}{s+1},
$$

so, in the notation of Eq. (2.5), the expansion coefficients are

$$
k_{s}=\frac{(-r)^{s}}{s+1} .
$$

Thus, for small $|r| a^{\prime}$,

$$
a=a^{\prime}\left[1-\frac{r a^{\prime}}{2}+O\left(\left(r a^{\prime}\right)^{2}\right)\right] .
$$

It follows that with the application of the $S_{L_{r}}$ scheme transformation,

$$
\begin{aligned}
& S_{L_{r}}: \quad a^{\prime}>a \text { if } r>0 \\
& a^{\prime}<a \text { if } r<0 .
\end{aligned}
$$

The requirement that the right-hand side of Eq. (3.1) be real implies that the argument of the log must be positive, which, in turn, yields the formal lower bound on this parameter

$$
r>-\frac{1}{a^{\prime}} .
$$

This is also required by the condition $C_{3}$, that the Jacobian must be (finite) and positive. If $r>0$, this inequality is obviously satisfied, since $a$ and $a^{\prime}$ are positive. Let us then consider negative $r$. Substituting Eq. (3.5), the inequality (3.11) becomes $r>r /\left(1-e^{r a}\right)$. Since we have restricted to negative $r$, this can be rewritten as $-|r|>-|r| /\left(1-e^{-|r| a}\right)$, i.e., $1<1 /\left(1-e^{-|r| a}\right)$, which is always satisfied. Thus, $r$ may be positive or negative, and the actual range of $r$ is determined by the conditions $C_{1}$ and $C_{2}$, that given a value of $\alpha=4 \pi a$ for which perturbative calculations are reasonably reliable, the same should be true of $\alpha^{\prime}=4 \pi a^{\prime}$. This will be discussed further below.

Substituting the result (3.8) for $k_{s}$ into the general expressions for the $b_{\ell}^{\prime}$ from [9], we obtain

$$
\begin{gathered}
b_{3}^{\prime}=b_{3}-\frac{r}{2} b_{2}-\frac{r^{2}}{12} b_{1}, \\
b_{4}^{\prime}=b_{4}-r b_{3}+\frac{r^{2}}{4} b_{2}+\frac{r^{3}}{12} b_{1}, \\
b_{5}^{\prime}=b_{5}-\frac{3 r}{4} b_{4}+\frac{5 r^{2}}{6} b_{3}-\frac{r^{3}}{8} b_{2}-\frac{13 r^{4}}{180} b_{1}, \\
b_{6}^{\prime}=b_{6}-2 r b_{5}+\frac{5 r^{2}}{3} b_{4}-\frac{2 r^{3}}{3} b_{3}+\frac{7 r^{4}}{120} b_{2}+\frac{11 r^{5}}{180} b_{1},
\end{gathered}
$$

and so forth for the $b_{\ell}^{\prime}$ with $\ell \geq 7$.

We next apply this $S_{L_{r}}$ scheme transformation to the beta function, in the $\overline{\mathrm{MS}}$ scheme, of an asymptotically free gauge theory. We take the gauge group to be $G=\mathrm{SU}(N)$. Since the $b_{\ell}$ have only been calculated up to $\ell=4$ loops in the $\overline{\mathrm{MS}}$ scheme, we will only need the results above for $b_{3}^{\prime}$ and $b_{4}^{\prime}$. For $N_{f} \in I$, so the two-loop $\beta$ function has an IR zero, we then calculate the resultant IR zero in $\beta_{\alpha^{\prime}}$ at the three- and four-loop order. We have carried out these calculations with a range of values of $N$ and $r$. For $N_{f} \in I$ and various values of $r$, we list the results for $N=3$, i.e., $G=\mathrm{SU}(3)$, in Table $\Pi$ for the zero of the three-loop beta function and in Table II for the zero of the four-loop beta function. We denote the IR zero of the $n$-loop beta function in the transformed scheme, $\beta_{\alpha^{\prime}, n \ell}$, as $\alpha_{I R, n \ell}^{\prime} \equiv \alpha_{I R, n \ell, S_{L_{r}}}^{\prime}$, and, to save space in the tables we further shorten this to $\alpha_{I R, n \ell, r}^{\prime}$. Here and below, for this $\mathrm{SU}(3)$ theory, the lower end of the interval $I$, namely $N=N_{f, b 2 z}$, is at $N=8.05$ [26], so, for physical, integral values of $N_{f}$, it is $N_{f}=9$. The lowest value we show in Table 1 and the later tables is $N_{f}=10$, because for $N_{f}=9, \alpha_{I R, 2 \ell}$ is too large for the perturbative methods that we use to be reliable. Our results for $N=2,4$, and other values are similar, so the $N=3$, i.e., $\mathrm{SU}(3)$ results displayed in Tables \and I will be sufficient for our discussion here. The range of $r$ for which we list results in these tables is $-3 \leq r \leq 3$. This range evidently satisfies the conditions $C_{1}-C_{4}$. For this range, the $S_{L_{r}}$ scheme transformation provides a useful quantitative measure of 
the scheme dependence of the IR zero in the beta function for this theory. Of course, if one were to increase the magnitude of $|r|$ to excessively large values, with either sign of $r$, this scheme transformation would not be useful, because it would violate conditions $C_{1}$ and $C_{2}$. For example, in the $\mathrm{SU}(3)$ theory with the illustrative value $N_{f}=12$, as one increases $r$ beyond the upper end of the range that we show, for the values $r=4,5,6$, 7, one gets the four-loop result $\alpha_{I R, 4 \ell, S_{L_{r}}}$ equal to $0.529,0.550$, $0.578,0.618$. But for $r=8$, the transformation yields a complex, unphysical result for $\alpha_{I R, 4 \ell, S_{L_{r}}}$. Similarly, for this $N_{f}=12$ case, as one decreases $r$ below the lowest negative value, $r=-3$, the solution for $\alpha_{I R, 4 \ell, S_{L_{r}}}$ decreases smoothly to 0.390 at $r=-10$, but becomes complex for $r=-11$. The resultant restriction on the range of the parameter $r$ is generic. Thus, as was discussed before in [8]-11], in applying scheme transformations, one must necessarily restrict the form of the transformation so as to satisfy the conditions $C_{1}-C_{4}$.

We also observe the following additional general properties in our calculations of $\alpha_{I R, n \ell, S_{L_{r}}}^{\prime}$. First, it follows from (2.15) together with the fact that Eq. (3.1) is a continuous transformation, that for small $|r|$, the relative order of the values of the $n$-loop IR zeros of $\beta_{\alpha^{\prime}}$ in the transformed scheme are the same as those in the original $\overline{\mathrm{MS}}$ scheme, as given in (2.14). This is evident from the illustrative $N=3$ results given in Tables $\llbracket$ and $\amalg$. In accord with (2.16), the shifts of the value of the IR zero as a function of loop order are larger for smaller $N_{f}$ and get smaller as $N_{f}$ approaches $N_{f, b 1 z}$.

Second, for a given $N, N_{f} \in I$, loop order $n=3$ or $n=4$, and $r$ values for which the $S_{L_{r}}$ transformation satisfies the conditions $C_{1}-C_{4}$,

$$
\alpha_{I R, n \ell, S_{L_{r}}}^{\prime} \text { is an increasing function of } r \text {. }
$$

This second property, in conjunction with the general property (2.15), implies that, for a given $N, N_{f} \in I$, and $r$,

$$
\begin{array}{ll}
\alpha_{I R, n \ell, S_{L_{r}}}^{\prime}>\alpha_{I R, n \ell, \overline{M S}} & \text { if } r>0 \text { and } \\
\alpha_{I R, n \ell, S_{L_{r}}}^{\prime}<\alpha_{I R, n \ell, \overline{M S}} & \text { if } r<0 .
\end{array}
$$

This holds for arbitrary loop order $n$ and, in particular, for the loop orders $n=3$ and $n=4$ for which we have done calculations using the known $\overline{\mathrm{MS}}$ beta function coefficients. The result (3.17) is evident in the illustrative $N=3$ results given in Tables [1 and II] In accord with (2.16), the shifts of the value of the IR zero as a function of $|r|$ become quite small as $N_{f}$ approaches $N_{f, b 1 z}$ from below. In this region, these shifts in the position of the IR zero of the $\ell$-loop beta function in the transformed scheme can be sufficiently small that the entries may coincide to the given number of significant figures displayed in the tables.

\section{THE RATIONAL SCHEME TRANSFORMATION $S_{[p, q]}$}

In [8]-11] a number of scheme transformations were studied for which the transformation function $f\left(a^{\prime}\right)$ has the form (2.5) with finite $s_{\max }$, i.e., is a (finite) polynomial in $a^{\prime}$. One way that it is possible to generalize these is to make $f\left(a^{\prime}\right)$ a rational function of $a^{\prime}$, i.e.,

$$
a=a^{\prime} f\left(a^{\prime}\right)_{[p, q]}
$$

with

$$
S_{[p, q]}: \quad f\left(a^{\prime}\right)_{[p, q]}=\frac{\mathcal{N}\left(a^{\prime}\right)}{\mathcal{D}\left(a^{\prime}\right)},
$$

where the numerator and denominator functions $\mathcal{N}\left(a^{\prime}\right)$ and $\mathcal{D}\left(a^{\prime}\right)$ are polynomials of respective finite degrees $p$ and $q$ in $a^{\prime}$ :

$$
\mathcal{N}\left(a^{\prime}\right)=\sum_{i=0}^{p} u_{i}\left(a^{\prime}\right)^{i} \quad \text { with } u_{0}=1
$$

and

$$
\mathcal{D}\left(a^{\prime}\right)=\sum_{j=0}^{q} v_{j}\left(a^{\prime}\right)^{j} \quad \text { with } v_{0}=1 .
$$

The restrictions that $u_{0}=v_{0}=1$ are imposed so that $f\left(a^{\prime}\right)$ satisfies the necessary condition that $f(0)=1$. Thus, a general $S_{[p, q]}$ scheme transformation depends on the $p+q$ parameters $u_{i}, i=1, \ldots, p$ and $v_{j}, j=1, \ldots, q$. As indicated, we label this class of scheme transformations as $S_{[p, q]}$, with the dependence on the coefficients $u_{i}$ and $v_{j}$ kept implicit. If $q=0$, then this gives a Taylor series expansion (2.5) of $f\left(a^{\prime}\right)_{[p, q]}$ with $s_{\max }=p$, while if $q \geq 1$, then $s_{\max }=\infty$.

We note that, as one may recall from the theory of Padé approximants, for a given series expansion (2.5) calculated to a given finite order $s_{h}$, it is possible to construct a set of rational functions $f\left(a^{\prime}\right)$ of the form (4.2) whose Taylor series expansion coefficients match the given set $k_{s}, s=1, \ldots, s_{h}$. Viewed the other way, if one starts with a set of rational functions of the form (4.2), one knows that certain subsets of these can be chosen to yield the same Taylor series expansion to a given order $s_{h}$.

The scheme transformation function $S_{[p, q]}$ introduces $p$ zeros and $q$ poles, so a necessary requirement is that one must choose the coefficients $u_{i}$ with $i=1, \ldots, p$ and $v_{j}$ with $j=1, \ldots, q$ such that the zeros and poles occur away from the relevant physical region in $a$. Obviously, scheme transformations with polynomial transformation functions $f\left(a^{\prime}\right)$ are special cases of $S_{[p, q]}$ with $q=0$. Thus, the scheme transformation $S_{1}$ studied in [8, 9] and [11] is a special case of $S_{[\underline{p}, q]}$ with $[p, q]=[1,0]$; the $S_{2}$ and $S_{3}$ transformations in $[8-10]$ are special cases of $S_{[p, q]}$ with $[p, q]=[2,0]$ and $[p, q]=[3,0]$, respectively; and the $S_{R, m}$ and $S_{R, m, k_{1}}$ transformations studied in [10, 11] are 
special cases of $S_{[p, q]}$ with $[p, q]=[m, 0]$. We proceed in the next section to study the simplest member of the class of $S_{[p, q]}$ scheme transformations with $q \neq 0$, namely the one with $[p, q]=[0,1]$.

\section{THE $S_{Q_{r}}$ SCHEME TRANSFORMATION}

In this section we introduce and apply a scheme transformation that we call $S_{Q_{r}}$, defined as $S_{[p, q]}$ with $[p, q]=$ $[0,1]$

$$
S_{Q_{r}} \equiv S_{[0,1]} \text { with } v_{1}=-r .
$$

Thus, explicitly,

$$
S_{Q_{r}}: \quad a=\frac{a^{\prime}}{1-r a^{\prime}},
$$

where $r$ is a (real) parameter, whose allowed range will be determined below. As before, we show this satisfies the necessary conditions to be acceptable at a zero of the beta function away from the origin for a reasonable range of $|r|$, and we then apply it to assess the scheme dependence of the IR zero in the beta function of an asymptotically free non-Abelian gauge theory at higher loop order. The transformation function corresponding to (5.2) is

$$
S_{Q_{r}}: \quad f\left(a^{\prime}\right)=\frac{1}{1-r a^{\prime}} .
$$

Clearly, $f\left(a^{\prime}\right)=1$ for $a^{\prime}=0$ and for separately for $r=0$. The inverse of Eq. (5.2) is

$$
a^{\prime}=\frac{a}{1+r a} .
$$

The Jacobian $J=d a / d a^{\prime}$ is

$$
J=\frac{1}{\left(1-r a^{\prime}\right)^{2}}=(1+r a)^{2} .
$$

The transformation function has the Taylor series expansion

$$
f\left(a^{\prime}\right)=1+\sum_{s=1}^{\infty}\left(r a^{\prime}\right)^{s},
$$

so, in the notation of Eq. (2.5), the expansion coefficients are

$$
k_{s}=r^{s} .
$$

Thus, for small $|r| a^{\prime}$,

$$
a=a^{\prime}\left[1+r a^{\prime}+O\left(\left(r a^{\prime}\right)^{2}\right)\right] .
$$

It follows that after application of the $S_{Q_{r}}$ scheme transformation,

$$
S_{Q_{r}}: \quad a^{\prime}<a \quad \text { if } r>0
$$

$$
a^{\prime}>a \text { if } r<0 .
$$

The condition $C_{1}$ requires that the denominator of the right-hand side of Eqs. (5.4) be finite and positive, which implies that the (real) parameter $r$ is bounded below according to

$$
r>-\frac{1}{a}
$$

Clearly, in order for conditions $C_{1}$ and $C_{2}$ to be satisfied, $r$ cannot be too close to saturating this lower bound. Applying these conditions to the original transformation (5.2) yields the formal inequality $r<1 / a^{\prime}$. However, substituting (5.4), this becomes $r<a^{-1}+r$, which is always valid, since $a>0$. Thus, the actual upper bound on $r$ is determined by the conditions $C_{1}$ and $C_{2}$, that, given a value of $\alpha$ for which perturbative calculations are reasonably reliable, the same should be true of $\alpha^{\prime}$.

Inserting the result (5.7) for $k_{s}$ into the general expressions for the $b_{\ell}^{\prime}$ from [9], we obtain

$$
\begin{gathered}
b_{3}^{\prime}=b_{3}+r b_{2}, \\
b_{4}^{\prime}=b_{4}+2 r b_{3}+r^{2} b_{2}, \\
b_{5}^{\prime}=b_{5}+3 r b_{4}+3 r^{2} b^{3}+r^{4} b_{2}, \\
b_{6}^{\prime}=b_{6}+4 r b_{5}+6 r^{2} b_{4}+4 r^{3} b_{3}+r^{4} b_{2},
\end{gathered}
$$

and so forth for the $b_{\ell}^{\prime}$ with $\ell \geq 7$. An important general property of these beta function coefficients resulting from the application of the $S_{Q_{r}}$ scheme transformation to an arbitrary initial scheme is that

$$
S_{Q_{r}}: \quad b_{\ell}^{\prime} \text { is independent of } b_{1} \text { for } \ell \geq 3 .
$$

The reason for this can be seen as follows. The coefficient $b_{\ell}^{\prime}$ with $\ell \geq 3$ resulting from the application of a scheme transformation is a linear combination of the $b_{n}$ with $1 \leq n \leq \ell$. The structure of the coefficients multiplying these $b_{n}$ with $1 \leq n \leq \ell$ was discussed in [8, 9]. In particular, the respective coefficients of $b_{1}$ in the expressions for $b_{\ell}^{\prime}$ with $\ell \geq 3$ have the property that they vanish if $k_{s}=\left(k_{1}\right)^{s}$. This property is satisfied by the present $S_{Q_{r}}$ scheme transformation, as is evident from Eq. (5.7). For example, in the expression (A1) for $b_{3}^{\prime}$ given in the Appendix, the coefficient of $b_{1}$ is $k_{1}^{2}-k_{2}$, and in Eq. A2 for $b_{4}^{\prime}$, the coefficient of $b_{1}$ is $-2 k_{1}^{3}+4 k_{1} k_{2}-2 k_{3}$; both of these coefficients of $b_{1}$ in $b_{3}^{\prime}$ and $b_{4}^{\prime}$ vanish if $k_{s}=\left(k_{1}\right)^{s}$. Similar results hold for the $b_{\ell}^{\prime}$ with higher values of $\ell$ that were calculated in [8]-[10].

We next apply this $S_{Q_{r}}$ scheme transformation to the beta function in the $\overline{\mathrm{MS}}$ scheme. We present results in Table III for the three-loop calculation and in Table IV for the four-loop calculation. The range of $r$ that we use is $-3 \leq r \leq 3$. For the lowest two values of $N_{f}$, 
namely $N_{f}=10$ and $N_{f}=11$, and the lowest values of $r$, namely $r=-3$, although the $S_{Q, r}$ scheme transformations yields acceptable values of the three-loop zero, $\alpha_{I R, 3 \ell, S_{Q_{r}}}^{\prime}$, it yields complex values of the four-loop zero, $\alpha_{I R, 4 \ell, S_{Q_{r}}}^{\prime}$. To avoid these, one may restrict the lower range of $r$ to, e.g., $r=-2$ for these values of $N_{f}$. The $S_{Q_{r}}$ transformation obeys the conditions $C_{1}$ and $C_{2}$ for positive values of $r$ somewhat beyond the upper end of the range that we show, but eventually, if one were to use excessively large values of $r$, it would again fail to satisfy these. We thus restrict the range of $r$ over which we apply this $S_{Q_{r}}$ scheme transformation accordingly.

We remark on some general features of the $S_{Q_{r}}$ scheme transformation. As with the $S_{L_{r}}$ transformation, it follows from (2.15) together with the fact that Eq. (5.2) is a continuous transformation, that for small $|r|$, the relative order of the values of the $n$-loop IR zeros of $\beta_{\alpha^{\prime}}$ in the transformed scheme are the same as those in the original $\overline{\mathrm{MS}}$ scheme, as given in (2.14). This is evident in Table III] and from Table IV] Second, for a given $N, N_{f} \in I$, and $r$, we find

$$
\begin{array}{ll}
\alpha_{I R, n \ell, S_{Q_{r}}}^{\prime}<\alpha_{I R, n \ell, \overline{M S}} & \text { if } r>0 \text { and } \\
\alpha_{I R, n \ell, S_{Q_{r}}}^{\prime}>\alpha_{I R, n \ell, \overline{M S}} & \text { if } r<0 \text { for } n=3,4 .
\end{array}
$$

Third, for a given $N, N_{f} \in I$, and loop order $n=3$ or $n=4$,

$$
\alpha_{I R, n \ell, S_{Q_{r}}}^{\prime} \text { is a decreasing function of } r .
$$

\section{COMPARATIVE DISCUSSION OF SCHEME TRANSFORMATIONS}

\section{A. $S_{s h_{r}}$ Scheme Transformation}

It is of interest to compare the $S_{L_{r}}$ and $S_{Q_{r}}$ scheme transformations with the the $S_{\mathrm{sh}_{\mathrm{r}}}$ scheme transformation studied in [8, 9] ,

$$
S_{\mathrm{sh}_{\mathrm{r}}}: \quad a=\frac{\sinh \left(r a^{\prime}\right)}{r} .
$$

Since $\sinh \left(r a^{\prime}\right) / r$ is an even function of $r$, one may take $r \geq 0$ without loss of generality. Equation (6.1) has the inverse

$$
a^{\prime}=\frac{1}{r} \ln \left[r a+\sqrt{1+(r a)^{2}}\right] .
$$

The corresponding transformation function is

$$
f\left(a^{\prime}\right)=\frac{\sinh \left(r a^{\prime}\right)}{r a^{\prime}},
$$

with expansion coefficients $k_{s}=0$ for odd $s$ and

$$
k_{2}=\frac{r^{2}}{6}, \quad k_{4}=\frac{r^{4}}{120}, \quad k_{6}=\frac{r^{6}}{5040},
$$

etc. for $s \geq 8$. Thus, for small $r a^{\prime}$,

$$
a=a^{\prime}\left[1+\frac{\left(r a^{\prime}\right)^{2}}{6}+O\left(\left(r a^{\prime}\right)^{4}\right)\right] .
$$

The Jacobian is

$$
J=\frac{d a}{d a^{\prime}}=\cosh \left(r a^{\prime}\right) .
$$

This Jacobian always satisfies condition $C_{3}$. From (6.4) or (6.6), it follows that $a^{\prime}<a$ for nonzero $r$ with this $S_{\mathrm{sh}_{\mathrm{r}}}$ scheme transformation.

\section{B. Comparative Discussion of Results with Different Scheme Transformations}

From the studies of a variety of scheme transformations in [8]- 11] and the present work, a number of general conclusions follow. These include the basic properties noted in Eqs. (2.15), (2.16), and the fact that for small $|r|$, the order of the values of the three-loop and four-loop IR zeros of the beta function are the same as in the $\overline{\mathrm{MS}}$ scheme, (2.14).

One basic property is that for values of the parameter(s) determining $f\left(a^{\prime}\right)$ (here, the parameter $r$ for the $S_{L_{r}}, S_{Q_{r}}$, and $S_{\mathrm{sh}_{\mathrm{r}}}$ transformations) such that $f\left(a^{\prime}\right)$ does not differ too much from the identity, the sign of the leading $k_{s}$ coefficient in the expansion (2.5) determines whether $a^{\prime}$ is greater or smaller than $a$. For the $S_{L_{r}}$ scheme transformation, this leading term for small positive $r$ is negative (cf. Eq. (3.1)), so $a^{\prime}>a$, while for the $S_{Q_{r}}$ and $S_{\mathrm{sh}_{\mathrm{r}}}$ scheme transformations, this leading term for small positive $r$ is positive (cf. Eqs. (5.2) and (6.5)), so $a^{\prime}<a$. Recall that with the $S_{\mathrm{sh}_{\mathrm{r}}}$ transformation, the leading term in the expansion (2.5) is the $k_{2}\left(a^{\prime}\right)^{2}$ term, while for the $S_{L_{r}}$ and $S_{Q_{r}}$ transformations, the leading term is $k_{1}\left(a^{\prime}\right)$.

In a similar manner, for a general scheme transformation $S_{r}$, the sign of the leading correction term in (2.5) also determines whether $\alpha_{I R, n \ell, S_{r}}^{\prime}$ is an increasing or decreasing function of $r$ for small $|r|$. Thus, the leading correction terms in $S_{L_{r}}$ scheme transformation is negative, and $\alpha_{I R, n \ell, L_{r}}^{\prime}$ is an increasing function of $r$, while for the the $S_{Q_{r}}$ and $S_{\mathrm{sh}_{\mathrm{r}}}$ scheme transformations, the leading correction term in (2.5) is positive, and $\alpha_{I R, n \ell, S_{Q_{r}}}^{\prime}$ and $\alpha_{I R, n \ell, S_{\mathrm{sh}} \mathrm{r}}^{\prime}$ are decreasing functions of $r$ and $|r|$, respectively $[36]$.

Concerning the range of $r$ over which a scheme transformation obeys the conditions $C_{1}-C_{4}$, we note that for the $S_{\mathrm{sh}_{\mathrm{r}}}$ transformation studied in [9], this range extended up to at least $|r|=4 \pi$, as was evident from the results displayed in Table III of [9]. Here, for the $S_{L_{r}}$ and also $S_{Q_{r}}$ scheme transformations, the respective allowed ranges of (positive and negative values of) $r$ are somewhat smaller. This is easily understood if one examines the Taylor series expansions of the respective transformation functions $f\left(a^{\prime}\right)$. The values of the coefficients $k_{s}$ with even $s$ (the odd-s ones being zero) for 
the $S_{\mathrm{sh}_{\mathrm{r}}}$ transformation in Eq. (6.4) are much smaller than those for the $k_{s}$ for both the $S_{L_{r}}$ and $S_{Q_{r}}$ transformations, listed, respectively, in Eqs. (3.8) and (5.7). Therefore, a given value of $r$ leads to a transformation function $f\left(a^{\prime}\right)$ that is considerably closer to the identity for the $S_{\mathrm{sh}_{\mathrm{r}}}$ scheme transformation than for the $S_{L_{r}}$ or $S_{Q_{r}}$ transformation. In general, if one constructs and applies a particular scheme transformation, one can see how large a deviation from the identity a moderate value of $r$ will produce for the transformation function $f\left(a^{\prime}\right)$ by examining the Taylor series expansion (2.5).

\section{SOME OTHER $S_{[p, q]}$ SCHEME TRANSFORMATIONS}

\section{A. $S_{[1,1]}$ Scheme Transformation}

In this section we remark on some other $S_{[p, q]}$ scheme transformations with $q \neq 0$. We begin with $S_{[1,1]}$, This is defined by the special case of (4.1) with $[p, q]=[1,1]$, namely

$$
S_{[1,1]}: \quad f\left(a^{\prime}\right)=\frac{1+u_{1} a^{\prime}}{1+v_{1} a^{\prime}},
$$

where $u_{1}$ and $v_{1}$ are (real) parameters. The inverse of Eq. (7.1) formally involves two solutions to a quadratic equation, but only one is physical, because it is the only one for which $a^{\prime} \rightarrow a$ as $\left(u_{1}, v_{1}\right) \rightarrow(0,0)$. This inverse transformation is

$$
a^{\prime}=\frac{-1+v_{1} a+\sqrt{\left(1-v_{1} a\right)^{2}+4 u_{1} a}}{2 u_{1}} .
$$

The Jacobian is

$$
J=\frac{1+2 u_{1} a^{\prime}+u_{1} v_{1}\left(a^{\prime}\right)^{2}}{\left(1+v_{1} a^{\prime}\right)^{2}} .
$$

The transformation function has a Taylor series expansion of the form (2.5) with

$$
k_{s}=\left(u_{1}-v_{1}\right)\left(-v_{1}\right)^{s-1} .
$$

\section{B. $S_{[1,2]}$ Scheme Transformation}

The $S_{[1,2]}$ scheme transformation is the special case of (4.1) with $[p, q]=[1,2]$, namely

$$
S_{[1,2]}: \quad f\left(a^{\prime}\right)=\frac{1+u_{1} a^{\prime}}{1+v_{1} a^{\prime}+v_{2}\left(a^{\prime}\right)^{2}},
$$

depending on the three (real) parameters $u_{1}, v_{1}$, and $v_{2}$. As with $S_{[1,1]}$, the inverse of (7.5) formally involves two solutions to a quadratic equation, but only one is physical because it is the only one for which $a^{\prime} \rightarrow a$ as $\left(u_{1}, v_{1}, v_{2}\right) \rightarrow(0,0,0)$. This inverse transformation is

$$
a^{\prime}=\frac{-1+v_{1} a+\sqrt{\left(1-v_{1} a\right)^{2}+4 a\left(u_{1}-v_{2} a\right)}}{2\left(u_{1}-v_{2} a\right)} .
$$

The Jacobian is

$$
J=\frac{1+2 u_{1} a^{\prime}+\left(u_{1} v_{1}-v_{2}\right)\left(a^{\prime}\right)^{2}}{\left(1+v_{1} a^{\prime}+v_{2}\left(a^{\prime}\right)^{2}\right)^{2}} .
$$

The transformation function has a Taylor series expansion of the form (2.5), but with coefficients $k_{s}$ that are more complicated than those for $S_{[0,1]}$ or $S_{[1,1]}$. The first few of these coefficients $k_{s}$ are

$$
k_{1}=\left(u_{1}-v_{1}\right)
$$

$$
\begin{gathered}
k_{2}=-\left(u_{1}-v_{1}\right) v_{1}-v_{2}, \\
k_{3}=\left(u_{1}-v_{1}\right) v_{1}^{2}+\left(2 v_{1}-u_{1}\right) v_{2},
\end{gathered}
$$

$$
k_{4}=-\left(u_{1}-v_{1}\right) v_{1}^{3}+\left(v_{2}-3 v_{1}^{2}+2 u_{1} v_{1}\right) v_{2},
$$

and so forth for higher $s$.

For sufficiently small $\left|u_{1}\right|$ and $\left|v_{1}\right|$, the $S_{[1,1]}$ scheme transformation obeys the conditions to be applicable at a (perturbatively calculated) IR zero of the beta function of an asymptotically free gauge theory. Similarly, for sufficiently small $\left|u_{1}\right|,\left|v_{1}\right|$, and $\left|v_{2}\right|$, the $S_{[1,2]}$ scheme also obeys these conditions. Because these scheme transformations involve two and three parameters, respectively, the analysis of the allowed ranges of these parameters is more complicated than the corresponding analyses given in $[8,10,11]$ and for the one-parameter scheme transformations $S_{L_{r}}$ and $S_{Q_{r}}$ here.

One could also consider $S_{[p, q]}$ scheme transformations with higher (finite) values of $p$ and/or $q$, but the inverses generically involve equations of cubic and higher degree, rendering the analytic calculations more cumbersome. We will thus not pursue these here.

\section{INFRARED-FREE THEORIES}

We have focused in this paper on the application of our scheme transformations $S_{L_{r}}$ and $S_{Q_{r}}$ to the study of the scheme dependence of the IR zero of the beta function in asymptotically free gauge theories. The question of scheme dependence also arises in studying the beta function to three loops and higher in an infrared-free theory, such as (in $d=4$ spacetime dimensions) (i) a $\mathrm{U}(1)$ gauge theory, (ii) a non-Abelian gauge theory with $N_{f}>N_{f, b 1 z}$ fermions in a given representation; and (iii) an $O(N)$ $\lambda|\vec{\phi}|^{4}$ scalar field theory. These IR-free theories have an IRFP at zero coupling, and one may search for a possible UV zero of the respective beta function. Again, it is straightforward to construct acceptable scheme transformations to apply in the vicinity of the IR fixed point of these theories at zero gauge or quartic scalar coupling, respectively, but considerably more difficult to do this when searching for a possible UV zero of the beta function (UVFP) away from the origin. Recently this search 
has been performed up to five-loop order in [23] for theories of type (i) and (ii) (see also [37]) and in [24] for theories of type (iii), with the finding of evidence against the existence of such a UVFP in these theories. Among other methods, these analyses made use of scheme transformations. Since these findings were quite robust, we have not deemed it necessary to apply the scheme transformations constructed here to these IR-free theories. An example of an IR-free theory that does exhibit such a UV zero (UVFP) was demonstrated from an exact solution of the $\mathrm{O}(N)$ nonlinear $\sigma$ model in $d=2+\epsilon$ dimensions in the $N \rightarrow \infty$ limit [38].

\section{CONCLUSIONS}

In this paper we have presented two new scheme transformations, $S_{L_{r}}$ and $S_{Q_{r}}$, and have used these to study the scheme-dependence of an infrared fixed point in an asymptotically free non-Abelian gauge theory, making comparison with the previous three-loop and four-loop calculations of the location of this point in the $\overline{\mathrm{MS}}$ scheme in [30,31]. Each of these scheme transformations depends on a parameter $r$, and we have shown that for a considerable range of values of $r$ in the two respective cases, for values of the scheme-independent two-loop IR zero of the beta function $\alpha_{I R, 2 \ell}$ that are sufficiently small that perturbative calculations are reasonably reliable, these scheme transformations introduce only relatively small shifts in the higher-loop values $\alpha_{I R, n \ell, S_{L_{r}}}$ and $\alpha_{I R, n \ell, S_{Q_{r}}}$, as compared with the respective $\alpha_{I R, n \ell, \overline{\mathrm{MS}}}$ for $n=3$ and $n=4$ loops. This agrees with and extends the results obtained with the $S_{\mathrm{sh}_{\mathrm{r}}}$ scheme transformation in [9] and also with the results of studies of different scheme transformations and specific schemes in [8]-[14]. Our results thus provide a further quantitative measure of the size of the scheme-dependence in the calculation of this fixed point at the three-loop and four-loop order, both at small and moderate couplings. We have also remarked on a generalized family of multi-parameter scheme transformations, $S_{[p, q]}$.

\section{Acknowledgments}

This research was partly supported by the NSF Grant No. NSF-PHY-13-16617. R. S. thanks T. A. Ryttov for valuable collaborative work on the related Refs. [8, 9] and 30,33$]$.

\section{Appendix A: Some Relevant Formulas}

In this appendix we include some relevant formulas used in the text. We first list the beta function coefficients $b_{\ell}^{\prime}$ calculated in [8, 9] that follow from a scheme transformation (2.4), as functions of $b_{n}$ in the original scheme. For our present analysis, we will use the threeloop and four-loop results [8, 9]

$$
b_{3}^{\prime}=b_{3}+k_{1} b_{2}+\left(k_{1}^{2}-k_{2}\right) b_{1},
$$

and

$$
b_{4}^{\prime}=b_{4}+2 k_{1} b_{3}+k_{1}^{2} b_{2}+\left(-2 k_{1}^{3}+4 k_{1} k_{2}-2 k_{3}\right) b_{1} .
$$

For our analysis of an interesting property of the $S_{Q_{r}}$ scheme transformation, we also display $b_{5}^{\prime}$ :

$$
\begin{aligned}
b_{5}^{\prime} & =b_{5}+3 k_{1} b_{4}+\left(2 k_{1}^{2}+k_{2}\right) b_{3}+\left(-k_{1}^{3}+3 k_{1} k_{2}-k_{3}\right) b_{2} \\
& +\left(4 k_{1}^{4}-11 k_{1}^{2} k_{2}+6 k_{1} k_{3}+4 k_{2}^{2}-3 k_{4}\right) b_{1} . \quad(\mathrm{A} 3)
\end{aligned}
$$

For a vectorial gauge theory with $N_{f}$ (massless) fermions transforming according to the representation $R$ of the gauge group $G$, the two scheme-independent coefficients in the beta function are [17]

$$
b_{1}=\frac{1}{3}\left(11 C_{A}-4 T_{f} N_{f}\right)
$$

and 18

$$
b_{2}=\frac{1}{3}\left[34 C_{A}^{2}-4\left(5 C_{A}+3 C_{f}\right) T_{f} N_{f}\right] .
$$

The calculations of [30], which are used as input for the present work, used $b_{3}$ and $b_{4}$ as calculated in the $\overline{\mathrm{MS}}$ scheme in [19, 20].
[1] Some early studies on the renormalization group include E. C. G. Stueckelberg and A. Peterman, Helv. Phys. Acta 26, 499 (1953); M. Gell-Mann and F. Low, Phys. Rev. 95, 1300 (1954); N. N. Bogolubov and D. V. Shirkov, Doklad. Akad. Nauk SSSR 103, 391 (1955); C. G. Callan, Phys. Rev. D 2, 1541 (1970); K. Symanzik, Commun. Math. Phys. 18, 227 (1970); K. Wilson, Phys. Rev. D 3, 1818 (1971).
[2] G. 't Hooft and M. Veltman, Nucl. Phys. B 44, 189 (1972). See also J. Ashmore, Lett. Nuovo Cimento 4, 289 (1972); C. G. Bollini and J. J. Giambiaggi, Nuovo Comento B 12, 20 (1972); and the early review G. Leibbrandt, Rev. Mod. Phys. 47, 849 (1975).

[3] G. 't Hooft, Nucl. Phys. B 61, 455 (1973).

[4] W. A. Bardeen, A. J. Buras, D. W. Duke, and T. Muta, Phys. Rev. D 18, 3998 (1978). 
[5] W. Celmaster and R. J. Gonsalves, Phys. Rev. D 20, 1420 (1979); E. Braaten and J. P. Leveille, Phys. Rev. D 24, 1369 (1981); J. A. Gracey, Phys.Lett. B 700, 79 (2011).

[6] S. J. Brodsky, G. P. Lepage, and P. B. Mackenzie, Phys. Rev. D 28, 228 (1983).

[7] M. Mojaza, S. J. Brodsky, and X.-G. Wu, Phys. Rev. Lett. 110, 192001 (2013) arXiv:1212.0049; S. J. Brodsky, M. Mojaza, and X.-G. Wu, Phys. Rev. D 89, 014027 (2014) arXiv:1304.4631; for a recent review, see X.-G. Wu, S. J. Brodsky, and M. Mojaza, Prog. Part. Nucl. Phys. 72, 44 (2013) arXiv:1302.0599 and references therein.

[8] T. A. Ryttov and R. Shrock, Phys. Rev. D 86, 065032 (2012) arXiv:1206.2366.

[9] T. A. Ryttov and R. Shrock, Phys. Rev. D 86, 085005 (2012), arXiv:1206.6895.

[10] R. Shrock, Phys. Rev. D 88, 036003 (2013) arXiv:1305.6524.

[11] R. Shrock, Phys. Rev. D 90, 045011 (2014) arXiv:1405.6244.

[12] T. A. Ryttov, Phys. Rev. D 89, 016013 (2014) arXiv:1309.3867.

[13] T. A. Ryttov, Phys. Rev. D 89, 056001 (2014) arXiv:1311.0848.

[14] T. A. Ryttov, Phys. Rev. D 90, 056007 (2014) arXiv:1408.5841.

[15] E. Brézin, J. C. Le Guillou, and J. Zinn-Justin, Phys. Rev. D 9, 1121 (1974).

[16] D. J. Gross, in R. Balian and J. Zinn-Justin, eds. Methods in Field Theory, Les Houches 1975 (North Holland, Amsterdam, 1976)

[17] D. J. Gross and F. Wilczek, Phys. Rev. Lett. 30, 1343 (1973); H. D. Politzer, Phys. Rev. Lett. 30, 1346 (1973); G. 't Hooft, unpublished.

[18] W. E. Caswell, Phys. Rev. Lett. 33, 244 (1974); D. R. T. Jones, Nucl. Phys. B 75, 531 (1974).

[19] O. V. Tarasov, A. A. Vladimirov, and A. Yu. Zharkov, Phys. Lett. B 93, 429 (1980); S. A. Larin and J. A. M. Vermaseren, Phys. Lett. B 303, 334 (1993).

[20] T. van Ritbergen, J. A. M. Vermaseren, and S. A. Larin, Phys. Lett. B 400, 379 (1997).

[21] G. 't Hooft, in The Whys of Subnuclear Physics, Proc. 1977 Erice Summer School, ed. A. Zichichi (Plenum, New York, 1979), p. 943.

[22] N. N. Khuri and O. A. McBryan, Phys. Rev. D 20, 881 (1979); A. V. Garkusha and A. L. Kataev, Phys. Lett. B 705, 400 (2011).

[23] R. Shrock, Phys. Rev. D 89, $045019 \quad$ (2014) arXiv:1311.5268.

[24] R. Shrock, Phys. Rev. D 90, 065023 (2014) arXiv:1408.3141.

[25] The Casimir invariants $C_{R}$ and $T_{R}$ are defined as $\sum_{a} \sum_{j} \mathcal{D}_{R}\left(T_{a}\right)_{i j} \mathcal{D}_{R}\left(T_{a}\right)_{j k}=C_{R} \delta_{i k}$ and $\sum_{i, j} \mathcal{D}_{R}\left(T_{a}\right)_{i j} \mathcal{D}_{R}\left(T_{b}\right)_{j i}=T_{R} \delta_{a b}$, where $R$ is the representation and $T_{a}$ are the generators of $G$, so that for $\mathrm{SU}\left(N_{c}\right), C_{A}=N_{c}$ for the adjoint $(A)$ and $T_{\text {fund }}=1 / 2$ for the fundamental representation, etc. $C_{f}$ denotes $C_{R}$ for the fermion representation.

[26] Here and elsewhere, when an expression is given for $N_{f}$ that formally evaluates to a non-integral real value, it is understood implicitly that one infers an appropriate integral value from it, either the greatest integer smaller than the real value, or the least integer greater than the real value, or the closest integer, depending on the context.

[27] T. Banks and A. Zaks, Nucl. Phys. B 196, 189 (1982).

[28] K. Yamawaki, M. Bando, and K. Matumoto, Phys. Rev. Lett. 56, 1335 (1986); T. Appelquist, D. Karabali, and L. C. R. Wijewardhana, Phys. Rev. Lett. 57, 957 (1986); V. A. Miransky, Dynamical Symmetry Breaking in Quantum Field Theories (World Scientific, Singapore, 1993).

[29] E. Gardi and M. Karliner, Nucl. Phys. B 529, 383 (1998); E. Gardi and G. Grunberg, JHEP 03, 024 (1999).

[30] T. A. Ryttov, R. Shrock, Phys. Rev. D 83, 056011 (2011) arXiv:1011.4542.

[31] C. Pica, F. Sannino, Phys. Rev. D 83, 035013 (2011) arXiv:1011.5917.

[32] For reviews, see, e.g., talks at the workshops LME-BSM (Lattice Meets Experiment: Beyond the Standard Model) 2012 at http://www-hep.colorado.edu/ schaich/lat-exp-2012 Lattice 2013 at http://www.lattice2013.uni-mainz.de; CP3 Workshop at http://cp3-origins.dk/events/meetings/mass2013 LME-BSM 2013 at http://www.bnl.gov/lme2013 and SCGT14 (Strongly Coupled Gauge Theories 2014)Mini at http://www.kmi.nagoya-u.ac.jp/workshop/SCGT14Mini and references therein.

[33] T. A. Ryttov and R. Shrock, Phys. Rev. D 85, 076009 (2012) arXiv:1202.1297.

[34] R. Shrock, Phys. Rev. D 87, 105005 (2013) arXiv:1301.3209; R. Shrock, Phys. Rev. D 87, 116007 (2013) arXiv:1302.5434.

[35] See, e.g., T. Appelquist and F. Sannino, Phys. Rev. D 59, 067702 (1999); S. Ignjatovic, L. C. R. Wijewardhana, and T. Takeuchi, Phys. Rev. D 61, 056006 (2000); M. Harada, M. Kurachi and K. Yamawaki, Phys. Rev. D 68, 076001 (2003); Phys. Rev. D 70, 033009 (2004); M. Kurachi and R. Shrock, JHEP 12, 034 (2006).

[36] In Eqs. (7.13) and (7.15) of Ref. [9], in the subscripts $S_{\mathrm{th}_{r}}$ should read $S_{\mathrm{sh}_{r}}$, as in Eq. (7.14) of Ref. [9].

[37] B. Holdom, Phys. Lett. B 694, 74 (2010) arXiv:1006.2119.

[38] W. A. Bardeen, B. W. Lee, and R. E. Shrock, Phys. Rev. D 14, 985 (1976); E. Brézin and J. Zinn-Justin, Phys. Rev. B 14, 3110 (1976); see also A. Polyakov, Phys. Lett. B 59, 79 (1975). 
TABLE I: Values of the IR zero, $\alpha_{I R, 3 \ell, S_{L_{r}}}^{\prime}$, of the three-loop beta function $\beta_{\alpha^{\prime}, 3 \ell}$ obtained by applying the $S_{L_{r}}$ scheme transformation to the three-loop beta function in the $\overline{\mathrm{MS}}$ scheme, for an $\mathrm{SU}(3)$ gauge theory with $N_{f}$ fermions in the fundamental representation. For compact notation, we set $\alpha_{I R, 3 \ell, S_{L_{r}}}^{\prime} \equiv \alpha_{I R, 3 \ell, r}^{\prime}$ in the table. For each $N_{f}$, we list these values as a function of $r$ for $r$ from $r=-3$ to $r=3$ in steps of 1 . For $r=0, \alpha_{I R, 3 \ell, S_{L_{r}}}^{\prime}=\alpha_{I R, 3 \ell, \overline{\mathrm{MS}}}$. We also list the (scheme-independent) two-loop IR zero of the beta function, $\alpha_{I R, 2 \ell}$

\begin{tabular}{|c|c|c|c|c|c|c|c|c|}
\hline \hline$N_{f}$ & $\alpha_{I R, 2 \ell}$ & $\alpha_{I R, 3 \ell, r=-3}^{\prime}$ & $\alpha_{I R, 3 \ell, r=-2}^{\prime}$ & $\alpha_{I R, 3 \ell, r=-1}^{\prime}$ & $\alpha_{I R, 3 \ell, \overline{M S}}$ & $\alpha_{I R, 3 \ell, r=1}^{\prime}$ & $\alpha_{I R, 3 \ell, r=2}^{\prime}$ & $\alpha_{I R, 3 \ell, r=3}^{\prime}$ \\
\hline 10 & 2.21 & 0.749 & 0.754 & 0.759 & 0.764 & 0.769 & 0.774 & 0.778 \\
11 & 1.23 & 0.566 & 0.570 & 0.574 & 0.578 & 0.583 & 0.587 & 0.591 \\
12 & 0.754 & 0.426 & 0.429 & 0.432 & 0.435 & 0.438 & 0.441 & 0.444 \\
13 & 0.468 & 0.311 & 0.313 & 0.315 & 0.317 & 0.319 & 0.321 & 0.323 \\
14 & 0.278 & 0.211 & 0.212 & 0.213 & 0.2145 & 0.216 & 0.217 & 0.218 \\
15 & 0.143 & 0.122 & 0.122 & 0.123 & 0.123 & 0.124 & 0.124 & 0.125 \\
16 & 0.0416 & 0.0396 & 0.0396 & 0.0397 & 0.0397 & 0.0398 & 0.0398 & 0.0399 \\
\hline \hline
\end{tabular}

TABLE II: Values of the IR zero, $\alpha_{I R, 4 \ell, S_{L_{r}}}^{\prime}$, of the four-loop beta function $\beta_{\alpha^{\prime}, 4 \ell}$ obtained by applying the $S_{L_{r}}$ scheme transformation to the four-loop beta function in the $\overline{\mathrm{MS}}$ scheme, for an $\mathrm{SU}(3)$ gauge theory with $N_{f}$ fermions in the fundamental representation. For compact notation, we set $\alpha_{I R, 4 \ell, S_{L_{r}}}^{\prime} \equiv \alpha_{I R, 4 \ell, r}^{\prime}$ in the table. For each $N_{f}$, we list these values as a function of $r$ for $r$ from $r=-3$ to $r=3$ in steps of 1 . For $r=0, \alpha_{I R, 4 \ell, S_{L_{r}}}^{\prime}=\alpha_{I R, 4 \ell, \overline{\mathrm{MS}}}$. We also list $\alpha_{I R, 2 \ell}$ and $\alpha_{I R, 3 \ell, \overline{\mathrm{MS}}}$.

\begin{tabular}{|c|c|c|c|c|c|c|c|c|c|}
\hline \hline$N_{f}$ & $\alpha_{I R, 2 \ell}$ & $\alpha_{I R, 3 \ell, \overline{\mathrm{MS}}}$ & $\alpha_{I R, 4 \ell, r=-3}^{\prime}$ & $\alpha_{I R, 4 \ell, r=-2}^{\prime}$ & $\alpha_{I R, 4 \ell, r=-1}^{\prime}$ & $\alpha_{I R, 4 \ell, \overline{M S}}$ & $\alpha_{I R, 4 \ell, r=1}^{\prime}$ & $\alpha_{I R, 4 \ell, r=2}^{\prime}$ & $\alpha_{I R, 4 \ell, r=3}^{\prime}$ \\
\hline 10 & 2.21 & 0.764 & 0.734 & 0.760 & 0.785 & 0.815 & 0.851 & 0.895 & 0.956 \\
11 & 1.23 & 0.578 & 0.576 & 0.591 & 0.607 & 0.626 & 0.648 & 0.673 & 0.705 \\
12 & 0.754 & 0.435 & 0.441 & 0.450 & 0.460 & 0.470 & 0.482 & 0.496 & 0.511 \\
13 & 0.468 & 0.317 & 0.322 & 0.327 & 0.332 & 0.337 & 0.343 & 0.349 & 0.356 \\
14 & 0.278 & 0.2145 & 0.217 & 0.219 & 0.221 & 0.224 & 0.226 & 0.228 & 0.231 \\
15 & 0.143 & 0.123 & 0.124 & 0.124 & 0.125 & 0.126 & 0.126 & 0.127 & 0.128 \\
16 & 0.0416 & 0.0397 & 0.0396 & 0.0397 & 0.0398 & 0.0398 & 0.0399 & 0.0400 & 0.0400 \\
\hline \hline
\end{tabular}


TABLE III: Values of the IR zero, $\alpha_{I R, 3 \ell, S_{Q_{r}}}$, of the three-loop beta function $\beta_{\alpha^{\prime}, 3 \ell}$ obtained by applying the $S_{Q_{r}}$ scheme transformation to the three-loop beta function in the $\overline{\mathrm{MS}}$ scheme, for an $\mathrm{SU}(3)$ gauge theory with $N_{f}$ fermions in the fundamental representation. For compact notation, we set $\alpha_{I R, 3 \ell, S_{Q_{r}}} \equiv \alpha_{I R, 3 \ell, r}^{\prime}$ in the table. For each $N_{f}$, we list these values as a function of $r$ for $r$ from $r=-3$ to $r=3$ in steps of 1 . For $r=0, \alpha_{I R, 3 \ell, S_{Q_{r}}}^{\prime}=\alpha_{I R, 3 \ell, \overline{\mathrm{MS}}}$. We also list the (scheme-independent) two-loop value of the IR zero, $\alpha_{I R, 2 \ell}$.

\begin{tabular}{|c|c|c|c|c|c|c|c|c|}
\hline \hline$N_{f}$ & $\alpha_{I R, 2 \ell}$ & $\alpha_{I R, 3 \ell, r=-3}^{\prime}$ & $\alpha_{I R, 3 \ell, r=-2}^{\prime}$ & $\alpha_{I R, 3 \ell, r=-1}^{\prime}$ & $\alpha_{I R, 3 \ell, \overline{M S}}$ & $\alpha_{I R, 3 \ell, r=1}^{\prime}$ & $\alpha_{I R, 3 \ell, r=2}^{\prime}$ & $\alpha_{I R, 3 \ell, r=3}^{\prime}$ \\
\hline 10 & 2.21 & 0.795 & 0.785 & 0.774 & 0.764 & 0.755 & 0.746 & 0.737 \\
11 & 1.23 & 0.605 & 0.596 & 0.587 & 0.5785 & 0.571 & 0.563 & 0.556 \\
12 & 0.754 & 0.455 & 0.448 & 0.441 & 0.435 & 0.429 & 0.423 & 0.418 \\
13 & 0.468 & 0.330 & 0.325 & 0.321 & 0.317 & 0.313 & 0.309 & 0.305 \\
14 & 0.278 & 0.222 & 0.219 & 0.217 & 0.215 & 0.212 & 0.210 & 0.208 \\
15 & 0.143 & 0.126 & 0.125 & 0.124 & 0.123 & 0.122 & 0.122 & 0.121 \\
16 & 0.0416 & 0.0401 & 0.0400 & 0.0398 & 0.0397 & 0.0396 & 0.0395 & 0.0394 \\
\hline \hline
\end{tabular}

TABLE IV: Values of the IR zero, $\alpha_{I R, 4 \ell, S_{Q_{r}}}^{\prime}$ of the four-loop beta function $\beta_{\alpha^{\prime}, 4 \ell}$ obtained by applying the $S_{Q_{r}}$ scheme transformation to the four-loop beta function in the $\overline{\mathrm{MS}}$ scheme, for an SU(3) gauge theory with $N_{f}$ fermions in the fundamental representation. For compact notation, we set $\alpha_{I R, 4 \ell, S_{Q_{r}}}^{\prime} \equiv \alpha_{I R, 4 \ell, r}^{\prime}$ in the table. For each $N_{f}$, we list these values as a function of $r$ for $r$ from $r=-3$ to $r=3$ in steps of 1 . For $r=0, \alpha_{I R, 4 \ell, S_{Q_{r}}}^{\prime}=\alpha_{I R, 4 \ell, \overline{\mathrm{MS}}}$. We also list $\alpha_{I R, 2 \ell}$ and $\alpha_{I R, 3 \ell, \overline{\mathrm{MS}}}$. The dash notation (-) means that the transformation yields an unphysical (here, complex) value for $\alpha_{I R, 4 \ell, S_{Q_{r}}}^{\prime}$.

\begin{tabular}{|c|c|c|c|c|c|c|c|c|c|}
\hline \hline$N_{f}$ & $\alpha_{I R, 2 \ell}$ & $\alpha_{I R, 3 \ell, \overline{\mathrm{MS}}}$ & $\alpha_{I R, 4 \ell, r=-3}^{\prime}$ & $\alpha_{I R, 4 \ell, r=-2}^{\prime}$ & $\alpha_{I R, 4 \ell, r=-1}^{\prime}$ & $\alpha_{I R, 4 \ell, \overline{M S}}$ & $\alpha_{I R, 4 \ell, r=1}^{\prime}$ & $\alpha_{I R, 4 \ell, r=2}^{\prime}$ & $\alpha_{I R, 4 \ell, r=3}^{\prime}$ \\
\hline 10 & 2.21 & 0.764 & - & 1.062 & 0.896 & 0.815 & 0.760 & 0.719 & 0.685 \\
11 & 1.23 & 0.578 & - & 0.750 & 0.674 & 0.626 & 0.591 & 0.563 & 0.540 \\
12 & 0.754 & 0.435 & 0.581 & 0.530 & 0.496 & 0.470 & 0.450 & 0.433 & 0.418 \\
13 & 0.468 & 0.317 & 0.380 & 0.363 & 0.349 & 0.337 & 0.327 & 0.318 & 0.309 \\
14 & 0.278 & 0.2145 & 0.239 & 0.233 & 0.228 & 0.224 & 0.219 & 0.215 & 0.211 \\
15 & 0.143 & 0.123 & 0.130 & 0.128 & 0.127 & 0.126 & 0.124 & 0.123 & 0.122 \\
16 & 0.0416 & 0.0397 & 0.0402 & 0.0401 & 0.0400 & 0.0398 & 0.0397 & 0.0396 & 0.0395 \\
\hline \hline
\end{tabular}

